\title{
Metastatic Carotid Body Tumor with Bivalvular Insufficiency in a Dog
}

\section{Dong-Yun Kim ${ }^{1, \dagger}$ \\ Jeong Uk Choi ${ }^{2, t}$ \\ Keon Kim ${ }^{1}$ \\ Sang-lk Park ${ }^{1}$ \\ Guk-Hyun Suh ${ }^{1}$ \\ Young-Chang $\mathrm{Cho}^{2, *}$ \\ Chang-Min Lee ${ }^{1, * *}$}

'Department of Veterinary Internal Medicine, College of Veterinary Medicine and BK21 FOUR Program, Chonnam National University, Gwangju 61186, Korea

${ }^{2}$ Research Institute of Pharmaceutical Sciences, College of Pharmacy, Chonnam National University, Gwangju 61186, Korea

'Dong-Yun Kim and Jeong Uk Choi contributed equally to this work.

*Correspondence: yccho@jnu.ac.kr (YoungChang Cho), cmlee1122@jnu.ac.kr (ChangMin Lee)

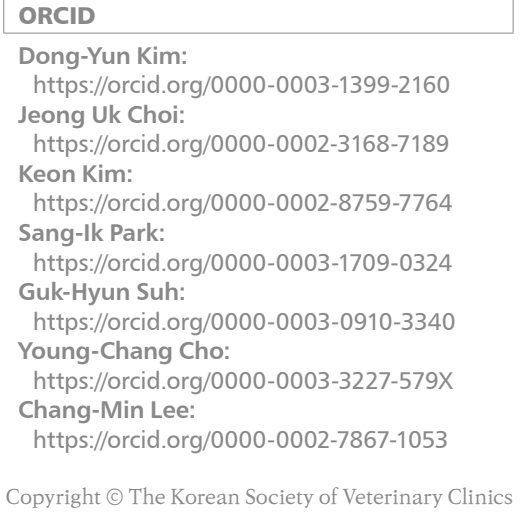

Abstract Compared to the other chemodectoma, aortic body tumors, the carotid body tumor is rarer and more often malignant. In the present case, a 12-year-old, intact female Shih-tzu dog presented to the hospital with a right ventral cervical mass. The mass was diagnosed by immunohistochemical staining with chromogranin A ( $\mathrm{CgA})$ as a carotid body tumor. The cervical mass and metastasized lymph node were removed by surgical resection. Because the dog had valvular heart disease, chemotherapy with carboplatin was initiated over 6 months to prevent metastasis and recurrence as an adjunctive treatment. After over two years of management, tumor metastasis and recurrence were not observed until recently. This report shows that proper management and chemotherapy as an adjunctive therapy can treat carotid body tumors in elderly dogs with concurrent heart disease.

Key words carboplatin, carotid body tumor, chemodectoma, dog, metastasis. 


\section{Introduction}

Generally, tumors of chemoreceptor organs are called paragangliomas or chemodectomas. These chemoreceptor organs include the aortic body and the carotid bodies which are responsible for detecting changes in carbon dioxide levels and blood oxygen, blood $\mathrm{pH}$, and blood pressure, respectively, thereby regulating respiration and circulation (6). The aortic bodies and carotid bodies are the common sites for the development of chemodectomas in dogs and cats (10). When compared the development of chemodectomas between animals and humans, in dogs, chemodectomas develop mainly in the aortic bodies, close to the heart base, and less commonly in the carotid bodies, located at the bifurcation of the common carotid arteries into internal and external carotid arteries; whereas in humans the carotid body tumor is the most common chemodectoma (10).

Radiation therapy has demonstrated value in isolated cases of human medicine (8), and a few chemotherapy treatments using different combinations of medicines have been reported $(1,3,7)$. Until recently, chemotherapy has not been evaluated in animals or humans as a primary or adjuvant therapy in the treatment of carotid body tumors (8). To date, it has been considered that surgical resection is typically the primary treatment of choice. As previously reported (5), there is only one case report in which carboplatin was used for systemic chemotherapy after surgical resection of a mass without metastasis. This report describes a case of a metastatic carotid body tumor in a dog managed by palliative chemotherapy using carboplatin.

\section{Case Presentation}

A 12-year-old, intact female Shih-tzu dog presented to the hospital with weight loss, depression, and decreased appetite. The patient's behavior included sitting all day long over the previous 3 months. Four days before presentation, a mass on the neck was found at the local hospital and tentatively diagnosed as a thyroid carcinoma. The heart murmur was auscultated and the dog was initiated with medical treatment for alleviating cardiovascular disorder. On the physical examination, tachycardia (heart rate: 150 bpm) and labored breathing with tachypnea (respiratory rate: $50 \mathrm{bpm}$ ) were detected, and the cause of respiratory distress was presumed to be due to mass compression. The body condition score was $2 / 5$ and the systolic blood pressure $(129 \mathrm{mmHg})$ was within the normal range. Pale mucous membranes were observed. There was a moveable, firm, round mass $36 \mathrm{~mm}$ in length and $24 \mathrm{~mm}$ in width near the cervical region (Fig. 1A). A systolic murmur of $3 / 6$ grade was auscultated in the mitral valve region. Cyanosis occurred easily when the dog was excited. The right prescapular lymph node (PSLN) was enlarged and there were mammary gland masses at 1st and 3rd left mammary glands. However, the size of the other lymph nodes was normal.

A radiographic examination was performed on the cervical, thoracic, and abdominal region. In the cervical exam, a mass at ventral and right to $\mathrm{C} 1-\mathrm{C} 4$ was noted. There was no displacement or narrowing of the trachea (Fig. 1B). In the thoracic radiography, cardiomegaly $(\mathrm{VHS}=10.7$, normal range is 9.2-10.2 in a small dog), left ventricle enlargement, and left atrium auricle bulging were observed. These results were likely due to aging and heart valve disease. In the abdominal exam, high radiopaque materials in bilateral kidneys with suspicion of renal calculi were detected.

An ultrasound examination was performed. A round, heterogeneous mass with vascular response in color doppler was detected, indicating a malignant tumor (Fig. 1C). The location of the mass was adjacent to the trachea and carotid artery, suggesting that it was likely a thyroid gland. An enlarged PSLN with suspected metastasis and without vascularity was observed. Echocardiography revealed mitral

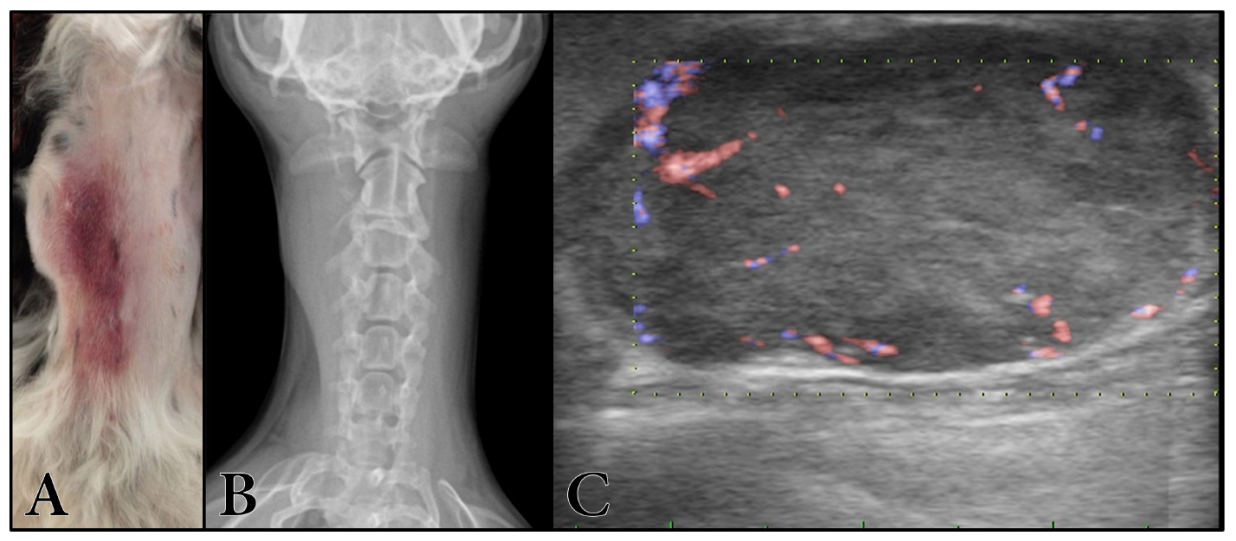

Fig. 1. The initial appearance, radiographic image and ultrasonographic image of the right ventral cervical mass. (A) The protrusion of the right cervical mass was grossly observed. Subcutaneous bleeding and redness of the cervical mass were noted. (B) Increased soft tissue opacity at the cervical region in the ventral view of the radiographic examination revealed the mass. (C) Heterogenous textured round mass with well margination was found. In color Doppler mode, vascular response was well imaged peripheral region of the mass. 
valve degeneration with increased preload [LVIDdN $=2.37$. There was no significant increase of left atrial pressure for preventative management. [E peak $=62.5 \mathrm{~cm} / \mathrm{s}$ ] The velocity of mitral valve regurgitation and tricuspid valve regurgitation were also measured in left apical view [MR flow PG $(\mathrm{mmHg})$ : 144.4, TR flow PG (mmHg): 28.9].

Fine needle aspiration (FNA) of the neck mass revealed vacuolated epithelial cells with anisokaryosis and anisocytosis, indicating a malignant tumor (Fig. 2A). Anisokaryosis, anisocytosis, and vacuolated epithelial cells were also observed in the PSLN. The neck and PSLN mass cytology patterns were similar. This suggested that the carcinoma was highly likely to metastasize (Fig. 2B). Although computerized tomography (CT) imaging was proposed for metastatic evaluation, the owner did not want to proceed because of the risks associat- ed with the anesthesia required for $\mathrm{CT}$ imaging.

As a result of performed examinations, bilateral renal calculi and right and left atrioventricular valvular insufficiency were detected by diagnostic imaging. Especially, mitral valve insufficiency could be classified as ACVIM stage B1. And metastatic thyroid carcinoma was tentatively diagnosed, which was required to differentiate from endocrine neoplasia for definitive diagnosis.

Based on the diagnosis, surgical removal and treatment were considered. In addition, management was necessary due to concurrent heart disease. Though no significant clinical signs were found, furosemide (1 $\mathrm{mg} / \mathrm{kg} B I D, P O)$ and ramipril $(0.125 \mathrm{mg} / \mathrm{kg} \mathrm{SID}, \mathrm{PO})$ were prescribed for the management of increased preload. After surgery, the ventral cervical mass and Rt. PSLN were removed. For histopatholog-
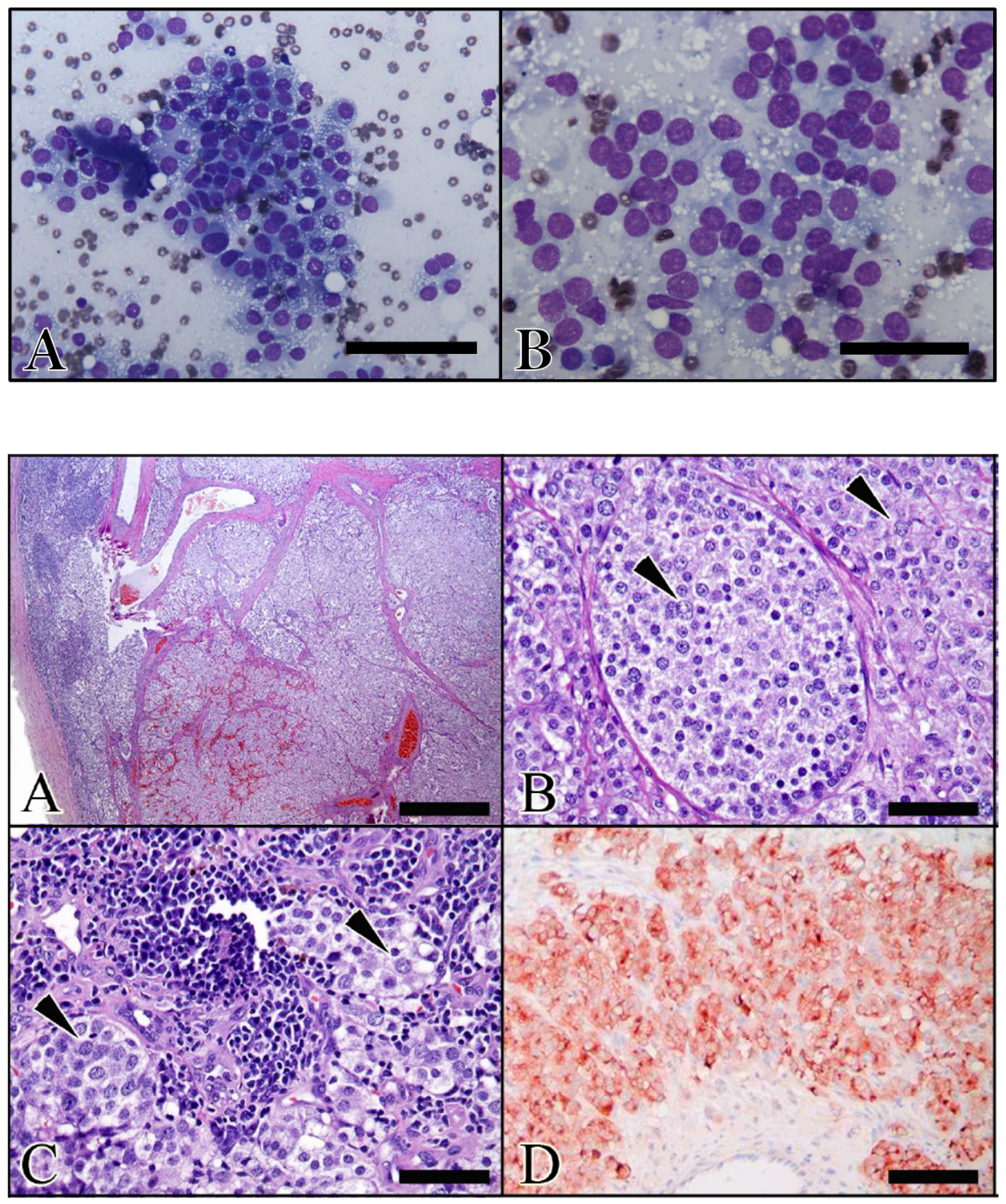

Fig. 2. Fine needle aspiration results of the cervical tumor and right prescapular lymph node. (A) The epithelial cells are crowded and stacked together, and have a high nucleus:cytoplasm ratio, in addition to moderate anisokaryosis ( $\times 40$ ). (B) A cellular pattern similar to that of the cervical tumor is observed $(\times 63)$.
Fig. 3. (A, B) Histopathological findings for tumor lesions in the cervical mass (H\&E, Bar $=500 \mu \mathrm{m}$, Bar $=80 \mu \mathrm{m}$ ) and (C) metastatic tumor lesion in the prescapular lymph node. Tumor cells are round to polyhedral, containing round-to-polyhedral, centrally located nuclei and distinct granular, lightly eosinophilic cytoplasm. Lymphatic metastasis is occurring (arrows) (H\&E, Bar $=50$ $\mu \mathrm{m})$. (D) Representative immunohistochemical staining for chromogranin-A (CgA) in the tumor lesions (Bar $=80 \mu \mathrm{m})$. 
ical examination, the tumor masses were sectioned ( $3 \mu \mathrm{m})$, and routinely stained with hematoxylin and eosin (H\&E). The selected sections were also subjected to immunohistochemistry $(\mathrm{IHC})$.

Microscopically, the tumor masses in the cervical region consisted of medium-sized cells arranged in nests separated by a fibrous stroma in the sinus, giving an endocrine-type packaging structures (Fig. 3A). The tumor cells were round to polyhedral, containing round-to-polyhedral nuclei and lightly eosinophilic cytoplasm with fine granules. They were highly pleomorphic with severe anisocytosis and anisokaryosis. However, neither formation of primitive follicular structures nor colloid-containing follicles formed by neoplastic follicular cells were found in multiple sections of tumor lesion. All together, these findings suggested characteristics of chemoreceptor tumors rather than those of thyroid tumors. Moreover, the same histopathological findings were observed in the tumor lesion in the prescapular lymph node (Fig. 3B).

IHC was performed to differentiate the tumors lesion in the cervical mass and prescapular region from thyroid tumors. The tumor cells were positive for chromogranin A (CgA) which is the maker for chemodectoma and neuroendocrine tumors (Fig. 3C). Moreover, tumor cells were negative for thyroglobulin and calcitonin, so thyroid tumors were under our least consideration. As a result, all these histopathological findings indicated that this case was of a malignant carotid body tumor.

After recovery of appetite and vitality, carboplatin (300 $\mathrm{mg} / \mathrm{m}^{2}$ IV q 21d) was administered for chemotherapy (over 3 weeks) due to the possibility of recurrence and other metastases, and metastasis to the distal lymph node was also confirmed. The medical treatment included Cephalexin (25 $\mathrm{mg} / \mathrm{kg} \mathrm{BID)}$, Ramipril (0.125 mg/kg SID), Furosemide (1 mg/kg BID), Misoprostol (5 $\mu \mathrm{g} / \mathrm{kg}$ BID), and Celecoxib (2 mg/kg BID). After medication, the pain symptoms decreased and appetite and vitality were improved.

The dog was subsequently treated with antineoplastic chemotherapy for 6 months to prevent metastasis. After 6 months, gastrointestinal side effects of the chemotherapy occurred and the chemotherapy discontinued. The treatment for heart disease has continued for over 2 years without recurrence and metastasis.

\section{Discussion}

A high incidence of bilateral or multicentric carotid body tumors in familial cases has not been reported dogs. However, carotid body tumors tend to be more malignant than aortic body tumors (8) and metastases have been found in about $30 \%$ of dogs $(9,10)$ in regional lymph nodes, lung, liver, pancreas, and kidney $(4,10)$. Metastases to the mediastinum and bones have also been reported, with an apparent predisposition for the vertebrae $(8,9)$. In this patient, the metastasis was confirmed by histopathology of the PSLN, and chemotherapy was performed after surgery due to the frequent metastasis of carotid body tumors.

Carotid body tumors usually cause clinical symptoms late in the course of the disease, since they exert a space occupying mass effect on the surrounding structures. Carotid body tumors tend to splay the carotid bifurcation as they enlarge (4), and treatment is difficult. In this case, pale mucous membrane and cyanosis upon excitation were observed suggesting mass effect as the cause. Since carotid body tumors are commonly not functional, they only have a mass effect due to size and location and the mass may press against the nearest artery, vein or the trachea. Consequently, blood and air-circulation (respiration) may be disturbed. In the current case, pale mucous membranes and cyanosis were observed upon excitation, likely due to the reasons described above. These clinical signs were no longer present after resection of the mass.

The cytologic features of thyroid carcinomas and carotid body tumors are not easily distinguished. In both tumors, the cells are ovoid, and occasionally have eccentrically placed nuclei with indistinct cytoplasmic borders. Moderate anisokaryosis and anisocytosis are remarkable features of malignancy in both tumors. FNA is easily contaminated by blood and the organs where tumors have developed are closely located. Therefore, it is difficult to distinguish those tumors by FNA features.

To differentiate a carotid body tumor from a thyroid tumor, histopathological analysis was performed. The characteristic morphological findings suggested a malignant carotid body tumor. Tumor lesions were subdivided into small compartments by fine septae plus small capillaries forming a classical neuroendocrine pattern (10). Round to polyhedral tumor cells arranged in distinct packets had lightly eosinophilic, finely granular, and often vacuolated cytoplasm. Ultrastructurally, the cytoplasm of chemodectoma as well as neuroendocrine tumors contain neurosecretory granules, which can be detected by antibody against chromogranin $\mathrm{A}(\mathrm{CgA})(2,10)$. Ectopic thyroid tumors are usually smaller than in aortic body tumors and have more hyperchromatic nuclei and eosinophilic cytoplasm (10). The neoplastic follicular cells are not consistently subdivided into small packet by fine strands of connective tissue and reticular fiber. In addition, no evidence for formation of thyroid follicle was found.

A doxorubicin-based protocol could be used in chemodec- 
toma, but alkylating agents such as cisplatin were considered instead because of the previously reported cardiotoxicity of doxorubicin (11). Also, cisplatin could cause nephrotoxicity and cardiac toxicity. Carboplatin is known for one of the alkylating agents that is similar to cisplatin but lower nephrotoxicity and cardiac toxicity. Therefore, carboplatin was selected considering that the dog had heart valvular disease and bilateral renal calculi. Furthermore, a half dose of carboplatin $\left(150 \mathrm{mg} / \mathrm{m}^{2} \mathrm{IV}\right)$ was administered compared to that of the most recommended due to the same reason.

There is one published report that is trying to treat with carboplatin (5), but this was administered for the completion of radiotherapy. To the author's knowledge, this is the first report concerning successful management of canine carotid body tumor without recurrence by using chemotherapy solely after surgical correction.

\section{Conclusions}

The dog in this case was successfully managed with chemotherapy using a carboplatin-based protocol for more than two years after surgical resection. The prognosis was considered guarded due to the presence of metastasis, concurrent heart disease, and old age. Nevertheless, neither recurrence of the carotid body tumor nor further metastasis was observed during over two years. This case report suggests that malignant carotid body tumors can effectively be managed with surgical resection and appropriate chemotherapy, even in cases of degenerative heart disease in elderly dogs.

\section{Acknowledgements}

This work was supported by the National Research Foundation of Korea (NRF) grant funded by the Korea government (MSIT) (No. 2019060984).

\section{Conflicts of Interest}

The authors have no conflicting interests.

\section{References}

1. Avgerinos ED, Moulakakis K, Brountzos E, Giannakopoulos TG, Lazaris AM, Koumarianou A, et al. Advances in assessment and management of carotid body tumors. Vascular 2011; 19: 250256.

2. Brown PJ, Rema A, Gartner F. Immunohistochemical characteristics of canine aortic and carotid body tumours. J Vet Med A Physiol Pathol Clin Med 2003; 50: 140-144.

3. Chrisoulidou A, Kaltsas G, llias I, Grossman AB. The diagnosis and management of malignant phaeochromocytoma and paraganglioma. Endocr Relat Cancer 2007; 14: 569-585.

4. Deim Z, Szalay F, Glávits R, Bauer A, Cserni G. Carotid body tumor in dog: a case report. Can Vet J 2007; 48: 865-867.

5. Dolera M, Carrara N, Malfassi L. VMAT stereotactic body radiation therapy in a multimodal approach to a carotid paraganglioma in a dog. J Am Anim Hosp Assoc 2018; 54: 111-116.

6. Eurell JA, Frappier BL, Dellmann HD. Dellmann's textbook of veterinary histology. 6th ed. Ames: Blackwell Publishing. 2006: 128129.

7. Huang H, Abraham J, Hung E, Averbuch S, Merino M, Steinberg SM, et al. Treatment of malignant pheochromocytoma/paraganglioma with cyclophosphamide, vincristine, and dacarbazine: recommendation from a 22-year follow-up of 18 patients. Cancer 2008; 113: 2020-2028.

8. Obradovich JE, Withrow SJ, Powers BE, Walshaw R. Carotid body tumors in the dog. Eleven cases (1978-1988). J Vet Intern Med 1992; 6: 96-101.

9. Okajima M, Shimada A, Morita T, Yoshikawa M, Nishida K. Multiple osseous metastases of a carotid body tumor in a dog. J Vet Med Sci 2007; 69: 297-299.

10. Rosol TJ, Meutens DJ. Tumors of the endocrine glands. In: Meuten DJ, editor. Tumors in domestic animals. 5th ed. Ames: John Wiley \& Sons Inc. 2017: 766-833.

11. Shi Y, Moon M, Dawood S, McManus B, Liu PP. Mechanisms and management of doxorubicin cardiotoxicity. Herz 2011; 36: $296-$ 305. 